\title{
CThU1.pdf
}

\section{Terahertz Technology in Outer and Inner Space}

\author{
Peter H. Siegel \\ California Institute of Technology and Jet Propulsion Laboratory \\ Pasadena, CA 91125 \\ phs@caltech.edu
}

SUMMARY:

After more than 30 years of niche applications in the space sciences area, the field of Terahertz Technology is entering a true Renaissance. While major strides continue to be made in submillimeter wave astronomy and spectroscopy, the past few years have seen an unprecedented expansion of terahertz applications, components and instruments. Broad popular interest in this unique frequency domain has emerged for the first time, spanning applications as diverse as biohazard detection and tumor recognition. Already there are groups around the world who have applied specialized Terahertz techniques to disease diagnostics, recognition of protein structural states, monitoring of receptor binding, performing label-free DNA sequencing and visualizing contrast in otherwise uniform tissue. A commercial terahertz imaging system has recently started tests in a hospital environment and new high sensitivity imagers with much deeper penetration into tissue have begun to emerge. Solicitations for more sophisticated instruments and enabling terahertz components have filtered into US agency proposal calls from DoD and NASA, to NSF and NIH, and many new research groups have sprung up, both in this country and in Europe and Asia. This presentation will broadly survey terahertz technology from its cradle applications in space science and spectroscopy to more recent biomedical and chemical uses.

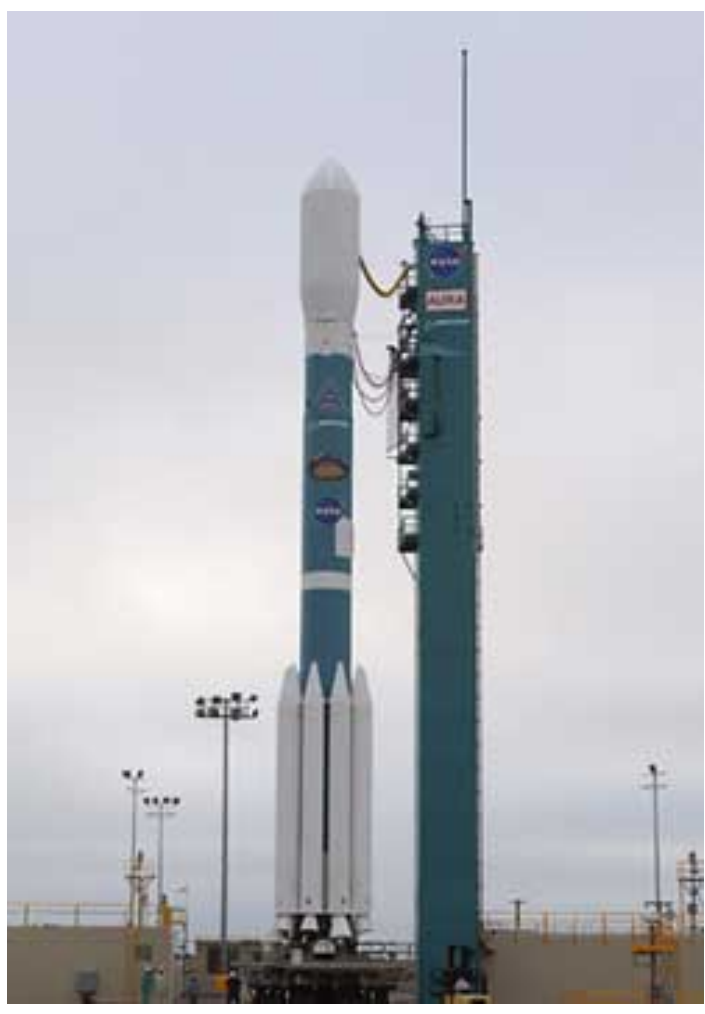

Aura spacecraft carrying Microwave Limb Sounder, a remote sensing stratospheric monitoring instrument with heterodyne receivers at $118,190,240,640$ and $2520 \mathrm{GHz}$.

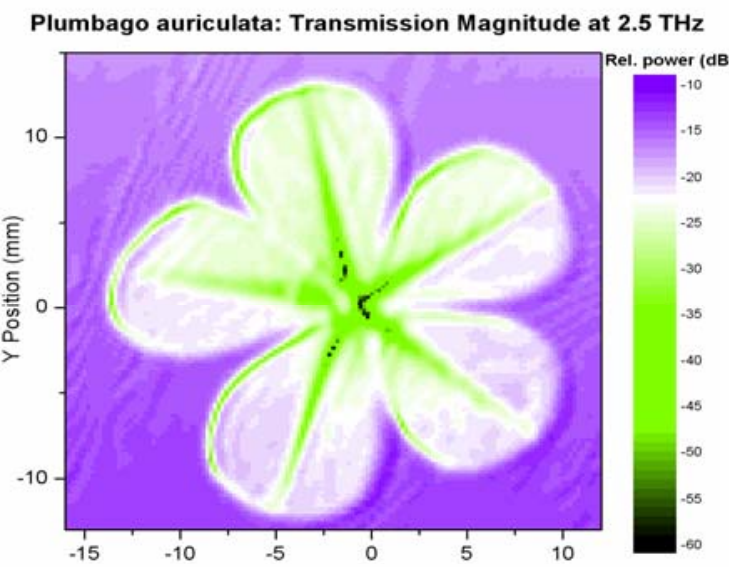

Plumbago auriculata: Transmission Phase at $2.5 \mathrm{THz}$

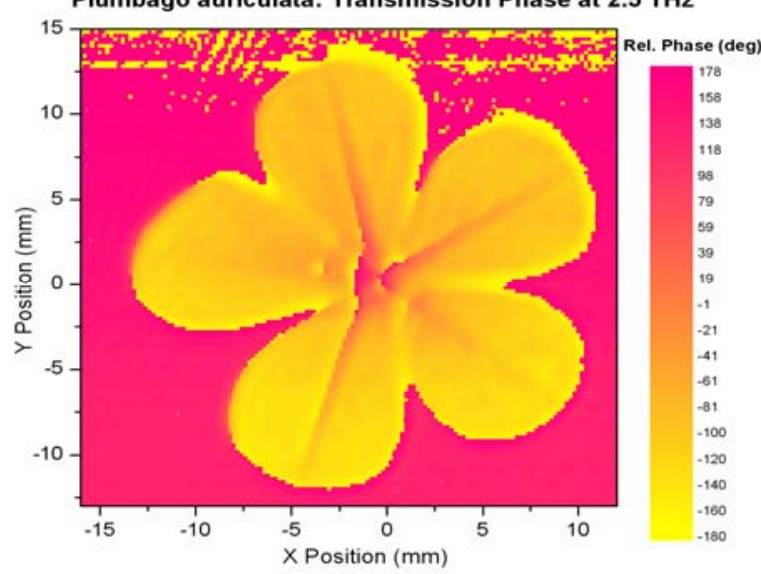

2.5 THz images of Plumbago auriculata, a local flower growing wild in the grounds of the Jet Propulsion Laboratory, Pasadena, California. Transmission magnitude (top) and phase (lower) images are shown in false color. Images were collected with a $2.5 \mathrm{THz}$ room temperature Schottky diode heterodyne radiometer and a strong $\mathrm{THz} \mathrm{CW}$ source, a $\mathrm{CO}_{2}$-pumped 